\title{
A Regression Model for Analysing the Non-linearity of Laser Triangulation Probes
}

\author{
Chensong Dong* \\ Department of Mechanical Engineering, Curtin University, GPO Box U1987, Perth, WA \\ 6845, Australia
}

\begin{abstract}
A study on the non-linearity of laser triangulation probes is presented in this paper. The influences of design variables on non-linearity were studied by Response Surface Methodology and the significant factors were identified. A regression model was developed, with which non-linearity can be conveniently evaluated in the design stage of laser triangulation probes. The sensitivities of non-linearity to the design variables are evaluated.
\end{abstract}

Keywords: Laser triangulation; non-linearity; regression

\section{Introduction}

Non-contact coordinate measurement is widely used today due to the demands of fast probing. Laser triangulation probes are dominant in the non-contact measurement on CMMs because of the advantages of simple structure and lightweight. However, the inherent non-linearity of laser triangulation measurement coming from the measurement principle limits its accuracy. Garcia et al. [1] used non-linear optical triangulation technique for the measurement of flatness. The non-linearity is corrected through calibration. Zhang et al. [2] presented a novel method using prism-based optical structure to correct the nonlinear problem of Laser Triangulation Displacement Measurement (LTDM). Power and Xue [3] introduced a technique that compensates for the real-world application by doing a system-wide nonlinear transform.

When studying the performance of laser triangulation probes, models are needed to simulate observed measurement errors. Smith and Zheng [4] developed a Point Laser Triangulation probe model to simulates observed measurement errors and shows the effects of placement and orientation of internal components.

In this paper, the non-linearity of a point laser triangulation probe was studied by Response Surface Methodology (RSM) using simulated data. A regression model was developed, with which the non-linearity can be conveniently evaluated in the design stage of laser triangulation probes. The sensitivities of non-linearity to the design variables are evaluated.

\section{Methodology}

A typical laser triangulation probe is as shown in Figure 1. The position of a target is

\footnotetext{
*Email: c.dong@curtin.edu.au
} 
determined by measuring reflected light from the target surface. A transmitter (laser diode) projects a light spot onto the target. In order to reduce the influence of measurand surface, the laser is projected perpendicularly $[5,6]$. The light is reflected off the target and focused via an optical lens system onto a light sensitive device built into the sensor head, called a receiving element. If the target changes its position from the reference point the position of the projected spot on the detector changes as well. The signal conditioning electronics of the laser detects the spot position on the position sensing device (PSD) and coupled with linearization and additional signal processing (digital or analogue) provides a proportional output signal (digital or analogue) to the position of the target.

PSD's are analogue detectors that rely on a current generated in a photodiode divided into one or two resistive layers. The amount of current from each output is proportional to the reflected light spot position on the detector. As shown in Figure 2, if the reflected light spot is in the middle of the detector the two analogue outputs will be equal and as it moves from the centre the two outputs change proportionally.

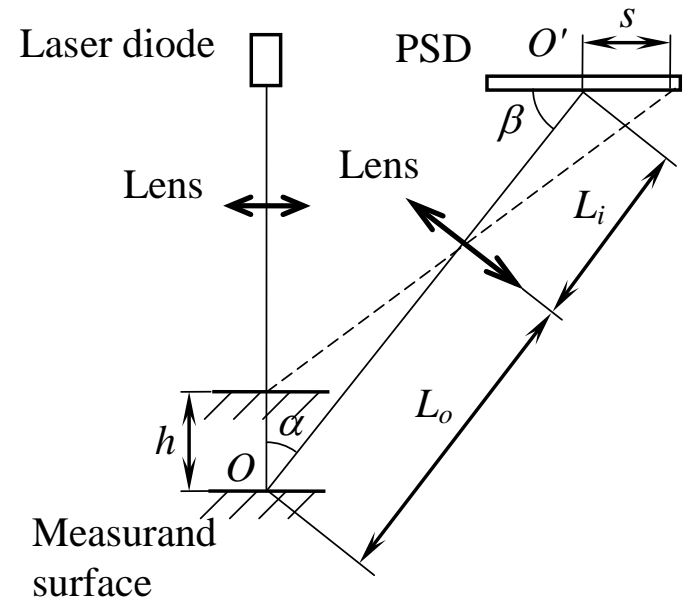

Figure 1: Laser triangulation probe

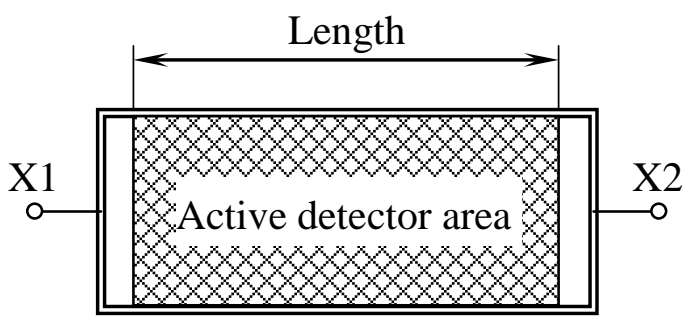

Spot position $=(\mathrm{X} 2-\mathrm{X} 1) /(\mathrm{X} 2+\mathrm{X} 1)$

Figure 2: Position sensing device

As shown in Figure 1, from geometric optics we have

$$
\frac{1}{L_{o}}+\frac{1}{L_{i}}=\frac{1}{f}
$$

where $L_{0}, L_{i}$ and $f$ are object distance, image distance and focal length, respectively.

It is derived from the similarity of triangles that

$$
\frac{h \sin \alpha}{s \sin \beta}=\frac{L_{o}-h \cos \alpha}{L_{i}+s \cos \beta}
$$

where $\alpha$ is optical angle and $\beta$ is PSD angle.

The height being measured is given by [7]

$$
h=\frac{L_{o} s \sin \beta}{L_{i} \sin \alpha+s \sin (\alpha+\beta)}
$$

Eqn. 3 suggests that the relationship between $h$ and $s$ is nonlinear. Although a linear 
relationship can be assumed when $h$ is very small, measurement errors are induced by this non-linearity.

In this study, Response Surface Methodology (RSM) was chosen to investigate the influence of design variables on non-linearity. Four design variables, $L_{o}, f, \quad \alpha$ and $\beta$ were chosen. $\quad L_{i}$ can be calculated from these four variables using Eqn. 1. For each variable, three levels were chosen. Thus, a $3^{4}$ factorial design was organised. The ranges of design variables were chosen based on a triangulation probe being developed in-house, which were dependent on the PSD and lenses being used, The ranges of design variables are-as shown in Table 1. The measurement range is $h= \pm 2 \mathrm{~mm}$.

Table 1: Design variables and levels

\begin{tabular}{lccc}
\hline Design variable & Low & Middle & High \\
\hline$L_{o}(\mathrm{~mm})$ & 30 & 40 & 50 \\
$f(\mathrm{~mm})$ & 10 & 15 & 20 \\
$\alpha\left(^{\circ}\right)$ & 30 & 45 & 60 \\
$\beta\left(^{\circ}\right)$ & 45 & 67.5 & 90 \\
\hline
\end{tabular}

For each variable combination, a series of $h$ vs. $s$ data was generated using Eqn. 3. A linear regression model was fitted to the data. For example, when $L_{o}=30 \mathrm{~mm}, f=10 \mathrm{~mm}, \alpha=$ $30^{\circ}$, and $\beta=45^{\circ}$, the $h$ vs. $s$ curve and the fitted linear model are shown in Figure 3. The linear model is $h=2.808 s-0.0609$. The error induced by non-linearity is shown in Figure 4.

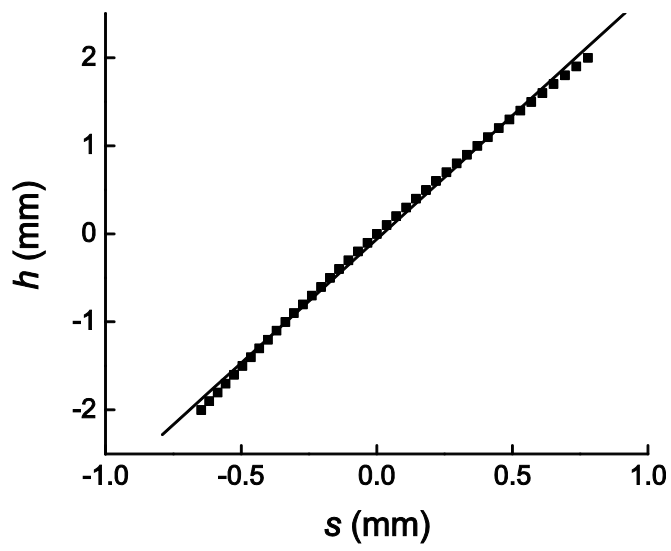

Figure 3: $h$ vs. $s$

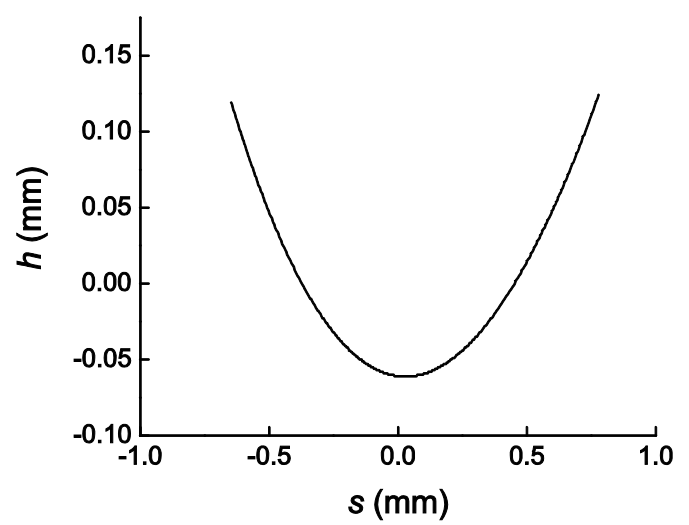

Figure 4: Error induced by non-linearity

In order to quantify non-linearity, degree of non-linearity $\left(d_{N L}\right)$ is introduced, which is defined as

$$
d_{N L}=\frac{S S E}{S S T}=1-\frac{S S R}{S S T}
$$

where SSE, SST, and SSR are the error sum squares, the total sum squares, and the regression sum squares, respectively [8]. A lower SSE implies the linear model provides a better fit to the data. In this case, $d_{N L}=0.22 \%$. 
After all $d_{N L}$ values were found, the Box-Cox transformation was conducted to normalise the data. This is a useful data (pre)processing technique used to stabilize variance, make the data more normal distribution-like, and improve the correlation between variables.

Logarithm transformation was chosen. The Analysis of Variance (ANOVA) suggests that a two factor interaction (2FI) model or a quadratic model gives similar accuracy. In order to keep the model simple, the 2FI model was chosen. Stepwise regression technique was used and it was found that $f$ was insignificant. The final regression model for $\ln \left(d_{N L}\right)$ is given by

$$
\begin{aligned}
\ln d_{N L} & =-0.291-4.56 \times 10^{-2} L_{o}+3.51 \times 10^{2} \alpha+6.50 \times 10^{-3} \beta \\
& -1.08 \times 10^{-4} L_{o} \beta-7.69 \times 10^{-4} \alpha \beta
\end{aligned}
$$

Non-linearity reaches its minimum when $L_{o}=50 \mathrm{~mm}, \alpha=60^{\circ}$ and $\beta=90^{\circ}$.

The sensitivities to the design variables are characterized by the coefficients of sensitivity $\left(C_{S}\right)$ as

$$
\begin{aligned}
C_{S L_{o}} & =\frac{\partial \ln d_{N L}}{\ln d_{N L} \cdot \partial L_{o}}=-4.56 \times 10^{-2}-1.08 \times 10^{-4} \beta \\
C_{S \alpha} & =\frac{\partial \ln d_{N L}}{\ln d_{N L} \cdot \partial \alpha}=3.51 \times 10^{2}-7.69 \times 10^{-4} \beta \\
C_{S \beta} & =\frac{\partial \ln d_{N L}}{\ln d_{N L} \cdot \partial \beta}=6.50 \times 10^{-3}-1.08 \times 10^{-4} L_{o}-7.69 \times 10^{-4} \alpha
\end{aligned}
$$

where $C_{S L o}, C_{S \alpha}$ and $C_{S \beta}$ are the coefficients of sensitivity to $L_{o}, \alpha, \beta$, respectively. $C_{S L o}, C_{S \alpha}$ and $C_{S \beta}$ are graphically shown in Figure 5 . It is seen that $C_{S \alpha}$ linearly decreases with increasing $\beta$; $C_{S L o}$ slightly decreases with increasing $\beta$; and $C_{S \beta}$ increases with $\alpha$, but slightly decreases with increasing $L_{o}$. The minimum sensitivities are reached when $L_{o}=30 \mathrm{~mm}, \alpha=$ $30^{\circ}$ and $\beta=45^{\circ}$.

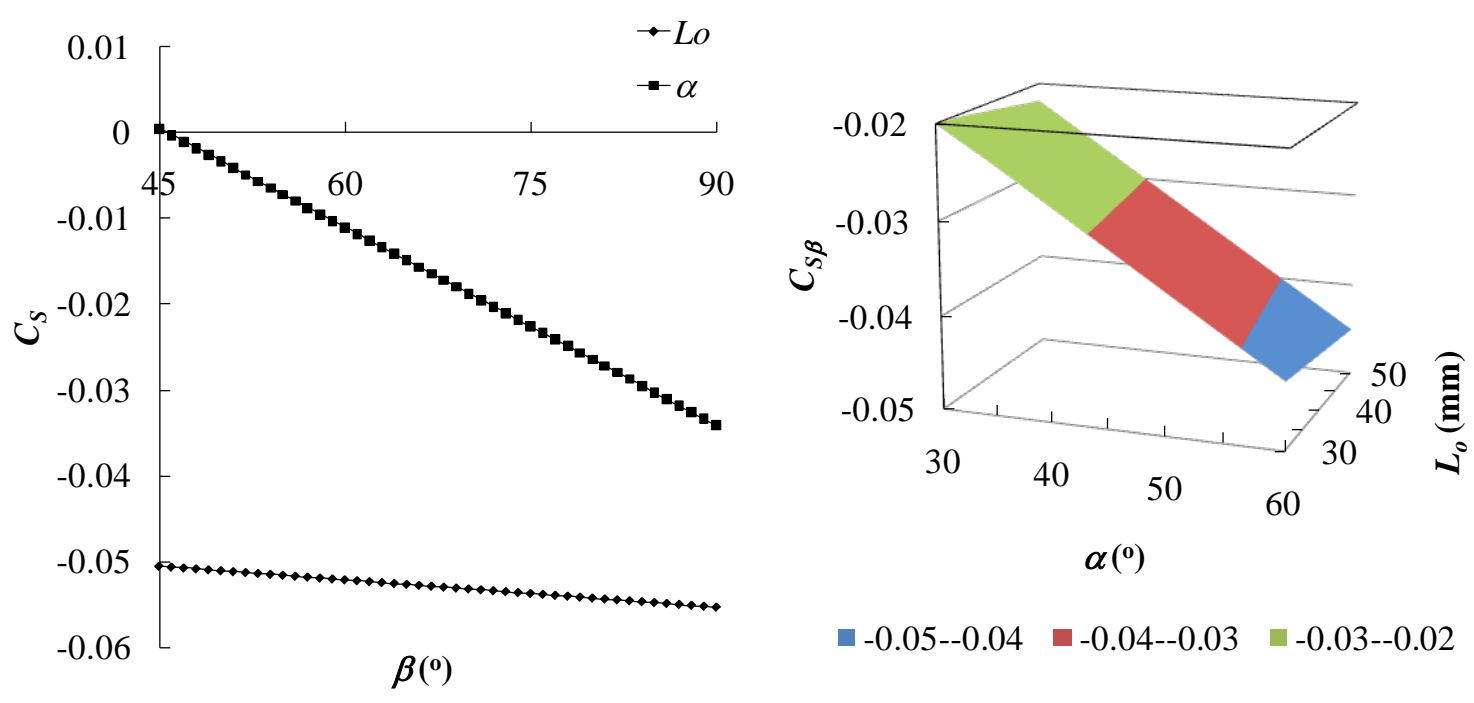

Figure 5: Coefficients of sensitivity 


\section{Conclusions}

A study on the non-linearity of laser triangulation probes is presented in this paper. The influences of design variables on non-linearity were studied by Response Surface Methodology and the significant factors were identified. A regression model was developed, with which non-linearity can be conveniently evaluated in the design stage of laser triangulation probes. It is seen that non-linearity decreases with increasing $L_{o}, \alpha$ or $\beta$, and non-linearity reaches its minimum when $L_{o}=50 \mathrm{~mm}, \alpha=60^{\circ}$ and $\beta=90^{\circ}$. The sensitivities of non-linearity to the design variables are evaluated. It is seen that $C_{S \alpha}$ linearly decreases with increasing $\beta$; $C_{S L o}$ slightly decreases with increasing $\beta$; and $C_{S \beta}$ increases with $\alpha$, but slightly decreases with increasing $L_{o}$. The minimum sensitivities are reached when $L_{o}=30$ $\mathrm{mm}, \alpha=30^{\circ}$ and $\beta=45^{\circ}$.

\section{References}

1. Garcia, D.F., et al., Flatness measurement system based on a nonlinear optical triangulation technique. IEEE Transactions on Instrumentation and Measurement, 2002. 51(2): p. 188 - 195.

2. Z Zhang, H., et al., Laser triangulation displacement measurement method Using prism-based optical structure, in 2008 IEEE Conference on Robotics, Automation and Mechatronics. 2008: Chengdu, China. p. 956 - 960.

3. Power, G.J. and K. Xue, A non-linear transform technique for a camera and laser 3-D scanner, in Proceedings of the IEEE 1995 National Aerospace and Electronics Conference (NAECON 1995). 1995 Dayton, OH, USA. p. 843 - 850.

4. $\quad$ Smith, K.B. and Y.F. Zheng, Accuracy analysis of Point Laser Triangulation probes using simulation. ASME Journal of Manufacturing Science and Engineering, 1998. 120(4): p. 736-745.

5. Kanada, H., Realization of a high accuracy laser displacement meter based on triangulation-reduction of work surface influence. Transactions of the Society of Instrument and Control Engineers, 1991. 27(8): p. 853-860.

6. Stevenson, W., The use of laser triangulation probes in coordinate measuring machines for part tolerance inspection and reverse engineering, in Proceedings of the SPIE - The International Society for Optical Engineering. 1992: Boston, MA, USA. p. 406-414.

7. Ji, Z. and M.C. Leu, Design of optical triangulation devices. Optics \& Laser Technology, 1989. 21(5): p. 339-341.

8. Montgomery, D.C., Design and Analysis of Experiments, 5th Edition. 5 ed. 2000, New York: John Wiley \& Sons, Inc. 
Appendix: Data Used for Regression

\begin{tabular}{|c|c|c|c|c|c|c|c|c|c|}
\hline $\begin{array}{c}L_{o} \\
(\mathbf{m m})\end{array}$ & $\underset{(\mathbf{m m})}{f}$ & $\begin{array}{c}\alpha \\
\left(^{\circ}\right)\end{array}$ & $\begin{array}{c}\beta \\
\left(^{\circ}\right)\end{array}$ & $\begin{array}{l}d_{N L} \\
(\%)\end{array}$ & $\begin{array}{c}L_{o} \\
(\mathbf{m m})\end{array}$ & $\underset{(\mathrm{mm})}{f}$ & $\begin{array}{c}\alpha \\
\left({ }^{\circ}\right)\end{array}$ & $\begin{array}{c}\beta \\
\left(^{\circ}\right)\end{array}$ & $\begin{array}{l}d_{N L} \\
(\%)\end{array}$ \\
\hline 30 & 10 & 30 & 45 & 0.22 & 40 & 15 & 45 & 90 & 0.03 \\
\hline 30 & 10 & 30 & 67.5 & 0.14 & 40 & 15 & 60 & 45 & 0.13 \\
\hline 30 & 10 & 30 & 90 & 0.09 & 40 & 15 & 60 & 67.5 & 0.05 \\
\hline 30 & 10 & 45 & 45 & 0.24 & 40 & 15 & 60 & 90 & 0.02 \\
\hline 30 & 10 & 45 & 67.5 & 0.12 & 40 & 20 & 30 & 45 & 0.13 \\
\hline 30 & 10 & 45 & 90 & 0.06 & 40 & 20 & 30 & 67.5 & 0.08 \\
\hline 30 & 10 & 60 & 45 & 0.22 & 40 & 20 & 30 & 90 & 0.05 \\
\hline 30 & 10 & 60 & 67.5 & 0.09 & 40 & 20 & 45 & 45 & 0.13 \\
\hline 30 & 10 & 60 & 90 & 0.03 & 40 & 20 & 45 & 67.5 & 0.07 \\
\hline 30 & 15 & 30 & 45 & 0.22 & 40 & 20 & 45 & 90 & 0.03 \\
\hline 30 & 15 & 30 & 67.5 & 0.14 & 40 & 20 & 60 & 45 & 0.13 \\
\hline 30 & 15 & 30 & 90 & 0.09 & 40 & 20 & 60 & 67.5 & 0.05 \\
\hline 30 & 15 & 45 & 45 & 0.24 & 40 & 20 & 60 & 90 & 0.02 \\
\hline 30 & 15 & 45 & 67.5 & 0.12 & 50 & 10 & 30 & 45 & 0.08 \\
\hline 30 & 15 & 45 & 90 & 0.06 & 50 & 10 & 30 & 67.5 & 0.05 \\
\hline 30 & 15 & 60 & 45 & 0.22 & 50 & 10 & 30 & 90 & 0.03 \\
\hline 30 & 15 & 60 & 67.5 & 0.09 & 50 & 10 & 45 & 45 & 0.09 \\
\hline 30 & 15 & 60 & 90 & 0.03 & 50 & 10 & 45 & 67.5 & 0.04 \\
\hline 30 & 20 & 30 & 45 & 0.22 & 50 & 10 & 45 & 90 & 0.02 \\
\hline 30 & 20 & 30 & 67.5 & 0.14 & 50 & 10 & 60 & 45 & 0.08 \\
\hline 30 & 20 & 30 & 90 & 0.09 & 50 & 10 & 60 & 67.5 & 0.03 \\
\hline 30 & 20 & 45 & 45 & 0.24 & 50 & 10 & 60 & 90 & 0.01 \\
\hline 30 & 20 & 45 & 67.5 & 0.12 & 50 & 15 & 30 & 45 & 0.08 \\
\hline 30 & 20 & 45 & 90 & 0.06 & 50 & 15 & 30 & 67.5 & 0.05 \\
\hline 30 & 20 & 60 & 45 & 0.22 & 50 & 15 & 30 & 90 & 0.03 \\
\hline 30 & 20 & 60 & 67.5 & 0.09 & 50 & 15 & 45 & 45 & 0.09 \\
\hline 30 & 20 & 60 & 90 & 0.03 & 50 & 15 & 45 & 67.5 & 0.04 \\
\hline 40 & 10 & 30 & 45 & 0.13 & 50 & 15 & 45 & 90 & 0.02 \\
\hline 40 & 10 & 30 & 67.5 & 0.08 & 50 & 15 & 60 & 45 & 0.08 \\
\hline 40 & 10 & 30 & 90 & 0.05 & 50 & 15 & 60 & 67.5 & 0.03 \\
\hline 40 & 10 & 45 & 45 & 0.13 & 50 & 15 & 60 & 90 & 0.01 \\
\hline 40 & 10 & 45 & 67.5 & 0.07 & 50 & 20 & 30 & 45 & 0.08 \\
\hline 40 & 10 & 45 & 90 & 0.03 & 50 & 20 & 30 & 67.5 & 0.05 \\
\hline 40 & 10 & 60 & 45 & 0.13 & 50 & 20 & 30 & 90 & 0.03 \\
\hline 40 & 10 & 60 & 67.5 & 0.05 & 50 & 20 & 45 & 45 & 0.09 \\
\hline 40 & 10 & 60 & 90 & 0.02 & 50 & 20 & 45 & 67.5 & 0.04 \\
\hline 40 & 15 & 30 & 45 & 0.13 & 50 & 20 & 45 & 90 & 0.02 \\
\hline 40 & 15 & 30 & 67.5 & 0.08 & 50 & 20 & 60 & 45 & 0.08 \\
\hline 40 & 15 & 30 & 90 & 0.05 & 50 & 20 & 60 & 67.5 & 0.03 \\
\hline 40 & 15 & 45 & 45 & 0.13 & 50 & 20 & 60 & 90 & 0.01 \\
\hline 40 & 15 & 45 & 67.5 & 0.07 & & & & & \\
\hline
\end{tabular}

